\title{
Prenatal Diagnosis of Dystrophin Gene Mutations using Multiplex Ligation Dependent Probe Amplification (MLPA) for Duchene Muscular Dystrophy
}

\author{
Asghar Nasir ${ }^{1}$, Zeeshan Ansar ${ }^{1}$, Shama Munim², Kahkashan Imam ${ }^{1}$ and Zahra Hasan*1 \\ ${ }^{1}$ Department of Pathology and Laboratory Medicine, Pakistan \\ ${ }^{2}$ Department of Obstetrics and Gynecology, Pakistan
}

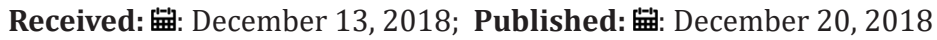

*Corresponding author: Zahra Hasan, Department of Pathology and Laboratory Medicine, Pakistan

\begin{abstract}
Duchene muscular dystrophy (DMD) is a common X-chromosomal recessive disorders caused by mutations in the dystrophin gene. Male children are primarily affected by the disease, characterized by progressive muscular wasting. The worldwide incidence of DMD is estimated at 1 in 3500 - 5000 male births. At present, there is no effective treatment for DMD, and there is a need for early diagnosis and pre-natal testing for dystrophin gene mutations. We describe the use of Multiple Probe Ligation and Amplification assay (MLPA) for diagnosis of dystrophin gene mutations in an amniocentesis sample tested at 16 weeks of gestation. The patient had a family history of DMD and also a child with DMD. In this case the sample was identified to be of a female fetus with a heterozygous deletion in dystrophin exons 49-50. Pre-natal diagnosis of DMD is an effective way for early identification of high risk cases suspected for DMD
\end{abstract}

Keywords: Duchene Muscular Dystrophy; Dystrophin Gene; Prenatal Testing; Carrier; Mutations

Abbreviations: DMD: Duchene Muscular Dystrophy; BMD: Becker Muscular Dystrophy; CVS: Chorion Villus Sampling; PCR: Polymerase Chain Reaction; MLPA: Multiplex Ligation-Dependent Probe Amplification; MCC: Multiplex Ligation-Dependent Probe Amplification

\section{Introduction}

Duchene muscular dystrophy (DMD) and Becker muscular dystrophy (BMD) are common X-chromosomal recessive disorders caused by mutations in the dystrophin gene. Male children are primarily affected by the disease, which is characterized by progressive muscular wasting and increased serum creatine kinase. The worldwide incidence of DMD is estimated to be about 1 in 3 , 500 to 1 in 5,000 male births [1]. In Pakistan, the incidence has not been reported, however few isolated studies have reported the gene mutation analysis patterns in the both distal and proximal hot spots in the effected patients [2-3]. DMD and BMD genotypes are characterized by mutations in the dystrophin gene, which has 79 exons. Up to $70 \%$ of DMD are associated with deletions, and $5-10 \%$ with duplications in the gene. However, approximately $25 \%$ to $30 \%$ of boys with DMD, and $5 \%$ to $10 \%$ of boys with BMD do not have whole exon deletions or duplications but have other small insertions/deletions/point mutations.
Genetic conditions are broadly classified into those which are either dominant or recessive and may be associated with either autosomes or sex-chromosomes. If the disease is X-linked, then a male individual with an $\mathrm{X}$ and $\mathrm{Y}$ chromosome with a mutation on the $\mathrm{X}$ chromosome would necessarily be affected by the disease condition. However, a female individual with the mutation may not be affected to the same extent as they would have a normal $\mathrm{X}$-chromosome to compensate for the mutation present. However, such a female individual who is heterozygous for the disease causing mutation may be termed as a 'carrier' for the disease [4]. As DMD is an X-linked disease, mothers with dystrophin gene mutations may be carriers who transfer risk for DMD to their off-spring [56]. Following that, female offspring may themselves be affected carriers while male offspring would definitely have DMD. Pre-natal testing performed during pregnancy can determine the genotype of the fetus. This allows the parents to make an informed decision 
to be prepared for a child with a likelihood for disease armed with knowledge that will help them to prepare and plan for the future or, to make a decision regarding the pregnancy outcome.

Different prenatal screening and diagnostic tests are possible. Chorion villus sampling (CVS) can be done at 10-12 weeks, and amniocentesis at about 14-16 weeks, while placental biopsy and fetal blood sampling can be done at about 18 weeks [7-8]. Currently the most widely used method for diagnosis of dystrophin gene mutation is based Multiplex Ligation-Dependent Probe Amplification (MLPA). In Multiple Probe Ligation and Amplification assay (MLPA), oligo-probes are hybridized to each dystrophin gene exon, followed by a ligation reaction. The ligated products are then amplified by polymerase chain reaction (PCR). Different length products are separated on an automated capillary sequencer where the amplified exon peaks are quantified. Deletions and duplications in the dystrophin gene are assessed by analysis software to determine the extent of mutations. Multiple Probe Ligation and Amplification assay (MLPA) is a powerful molecular tool for the detection of deletions or duplication in any of the 79 exons in the dystrophin gene. The test employed is a MLPA kits, SALSA P034-B1 and P035-B1 (MRC-Holland, Netherlands, CE-IVD marked) which can identify deletion/duplication mutations in 79 exons of dystrophin gene.

\section{Case Report}

We report a case in which a 24 years old lady gravida 2 and para 1+0 referred from Aga Khan Hospital Hyderabad for prenatal diagnosis in November 2017 to the Fetal Medicine Unit, Department of Obstetrics and Gynecology, The Aga Khan University, Karachi. The study subject presented at 12 weeks of gestation and had a previous history of a three-year-old male child affected by DMD. She was advised for pre-natal diagnosis of dystrophin gene mutation testing for which amniocentesis was performed at $16+1$ weeks of gestation. In addition, a blood sample was taken from the study subject and also her son affected by DMD. Blood and amniocentesis specimens were submitted to the Section of Molecular Pathology, The Aga Khan University Clinical Laboratory for Dystrophin gene mutation testing by MLPA. First, a check for maternal cell contamination (MCC) was performed in the pre-natal sample in order to ensure that the diagnostic result would be robust and not likely to be influenced by carryover of any maternal DNA.

Figure 1 depicts the results obtained and reveals a heterozygous deletion in Exons 49-50 of dystrophin gene to be present in ' $A$ ' the pre-natal specimen and the ' $\mathrm{B}$ ' the female patient. In addition, a deletion of dystrophin exons 49-50 was observed in the male child affected by DMDM ' $\mathrm{C}$ '. Overall, the results indicated that the fetus, like her mother, would be a female carrier positive for dystrophin gene mutations. Also, that the affected male child had acquired the same mutation from his mother. Based on this result, the patient was referred back to her home town Hyderabad, Sindh, for further antenatal care. Unfortunately, the study subject did not have the recommended anomaly scan done at 20 weeks of gestation. Instead, a growth ultrasound was performed at 27 weeks of gestation which showed the evidence of hydrops fetalis. Due to this result, the family opted to discontinue the pregnancy and termination of pregnancy was performed at 28 weeks of gestation.

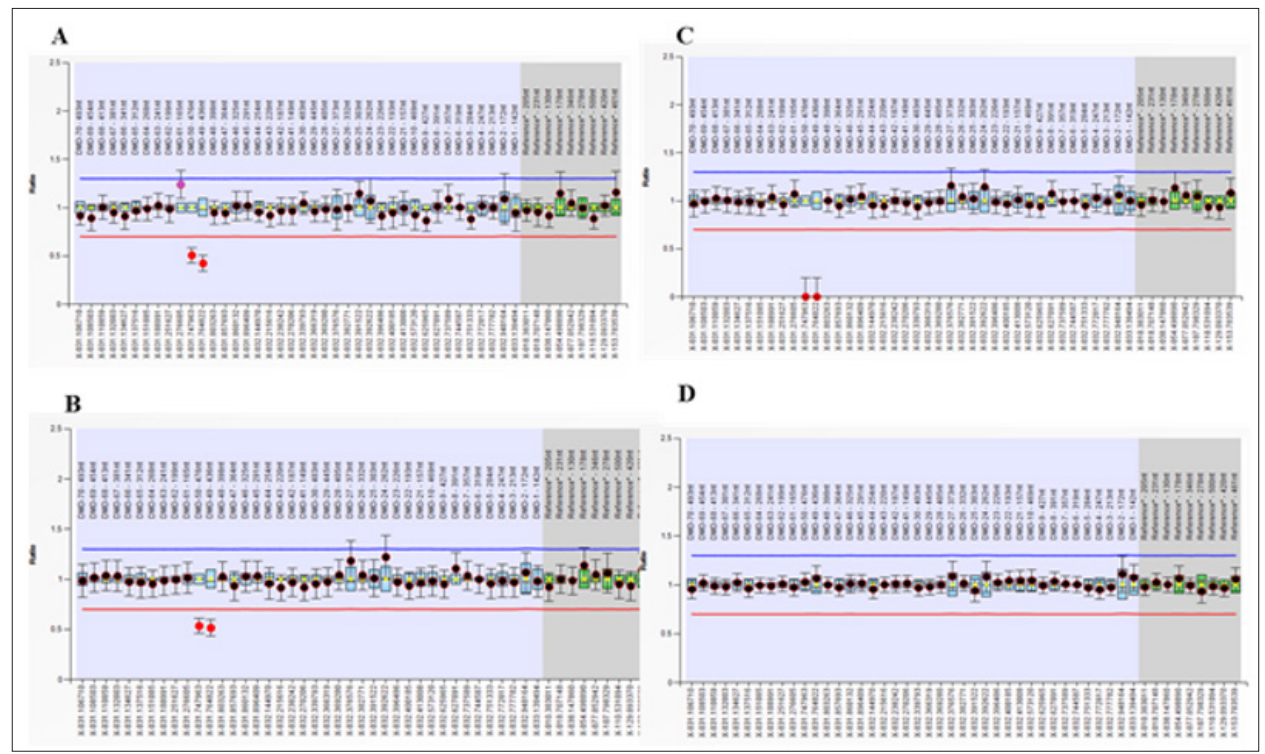

Figure 1: Detection of mutations in dystrophin gene Exons by MLPA. MLPA kits, SALSA P034-B1 and P035-B1 (MRC-Holland, Netherlands), were used to detect the deletion/duplication mutations in 79 exons of dystrophin gene in the Amnio, patient, DMD affected boy of the patient, and normal healthy control sample according to the instructions of MLPA kits.

A, B: MLPA SALSA P034-B1 showed a dosage quotient (DQ) ratio of 0.5 in Exon 49-50 (heterozygous deletion) in both the pre-natal sample and the study subject.

C: The DQ ratio 0 for Exon 49-50 (homozygous deletion) was observed in the sample for DMD affected child.

D: Normal healthy control sample had a DQ ratio 1 for all Exon, no deletion or duplication was detected. 


\section{Discussion}

DMD is a progressive genetic disorder. It an X-linked condition caused by a mutation in the dystrophin gene. This gene encodes the dystrophin protein and lies on the short arm of chromosome $\mathrm{X}$ (Xp21.2-p21.1). Mutations in this gene also give rise to a milder form of muscular dystrophy, with later onset, called Becker Muscular Dystrophy (BMD). Various mutations can give rise to DMD, but the most common are large deletions of sections of the gene, which are reported to account for two thirds of cases. About $61 \%$ of cases are caused by deletions, $13 \%$ by duplications, and $26 \%$ by point mutations or other small mutations. The deletions that give rise to DMD are often those that result in a frame shift, i.e. deletions that cause all the subsequent sections of the gene to be read incorrectly by the protein making machinery of the cell.

\section{Conclusion}

MLPA for dystrophin gene mutations revealed that amnio exhibited heterozygous deletion in Exon 49-50, same as observed that in the mother. The effected boy exhibited a homozygous deletion in Exon 49-50. Women have two X chromosomes, and therefore if they have only one $\mathrm{X}$ chromosome with a mutation in the dystrophin gene, the other copy of the gene may be able to compensate. Once a boy is identified as being affected by DMD or BMD, their mother should be assessed to see if she also carries the mutation [9-10]. Given that there is no available treatment, the greatest benefits of screening would be that a very small number of births of second affected boys would be averted. In the absence of a cure, prenatal diagnosis appears to be the best approach to reduce the burden of this disease on the individual family and ultimately on society.

\section{Acknowledgement}

This study received approval from the Ethical Review Committee, The Aga Khan University, Pakistan.

\section{ISSN: 2574-1241}

DOI: $10.26717 / B J S T R .2018 .12 .002251$

Zahra Hasan. Biomed J Sci \& Tech Res

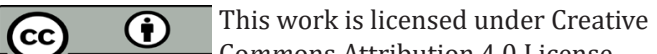

This work is licensed under Creative
Commons Attribution 4.0 License

Submission Link: https://biomedres.us/submit-manuscript.php

\section{References}

1. Mah JK, Korngut L, Dykeman J, Day L, Pringsheim T, et al. (2014) A systematic review and meta-analysis on the epidemiology of Duchenne and Becker muscular dystrophy. Neuromuscular disorders 24(6): 482491.

2. Hassan MJ, Mahmood S, Ali G, Bibi N, Waheed I, et al. (2008) Intragenic deletions in the dystrophin gene in 211 Pakistani Duchenne muscular dystrophy patients. Pediatrics International 50(2): 162-166.

3. Rahim RS, Jamil A, Rehman A, Sheikh MA, Jamil A (2017) Molecular analysis of Duchenne Muscular Dystrphy (DMD) in Patients and Carriers. International Journal of Advances in Science Engineering and Technology 5(3).

4. Lee SH, Lee JH, Lee KA, Choi YC (2015) Clinical and genetic characterization of female dystrophinopathy. Journal of Clinical Neurology 11(3): 248-251.

5. Mercier S, Toutain A, Toussaint A, Raynaud M, De Barace C, et al. (2013) Genetic and clinical specificity of 26 symptomatic carriers for dystrophinopathies at pediatric age. European Journal of Human Genetics 21(8): 855.

6. Brioschi S, Gualandi F, Scotton C, Armaroli A, Bovolenta M, et al. (2012) Genetic characterization in symptomatic female DMD carriers: lack of relationship between X-inactivation, transcriptional DMD allele balancing and phenotype. BMC medical genetics 13(1): 73.

7. Gatheridge MA, Kwon JM, Mendell JM, Scheuerbrandt G, Moat SJ, et al. (2016) Identifying non-Duchenne muscular dystrophy-positive and false negative results in prior Duchenne muscular dystrophy newborn screening programs: a review. JAMA neurology 73(1): 111-116.

8. Yoo SK, Lim BC, Byeun J, Hwang H, Kim KJ, et al. (2015) Noninvasive prenatal diagnosis of duchenne muscular dystrophy: comprehensive genetic diagnosis in carrier, proband, and fetus. Clinical chemistry 61(6): 829-837.

9. Nozoe KT, Akamine RT, Mazzotti DR, Polesel DN, Grossklauss LF, et al. (2016) Phenotypic contrasts of Duchenne Muscular Dystrophy in women: Two case reports. Sleep Science 9(3): 129-133.

10. Li T, Zhang ZJ, Ma X, Lv X, Xiao H, et al. (2017) Prenatal diagnosis for a Chinese family with a de novo DMD gene mutation: A case report. Medicine 96(50): 8814.

Submission Link: htps://biomedres.us/submit-manuscript.php

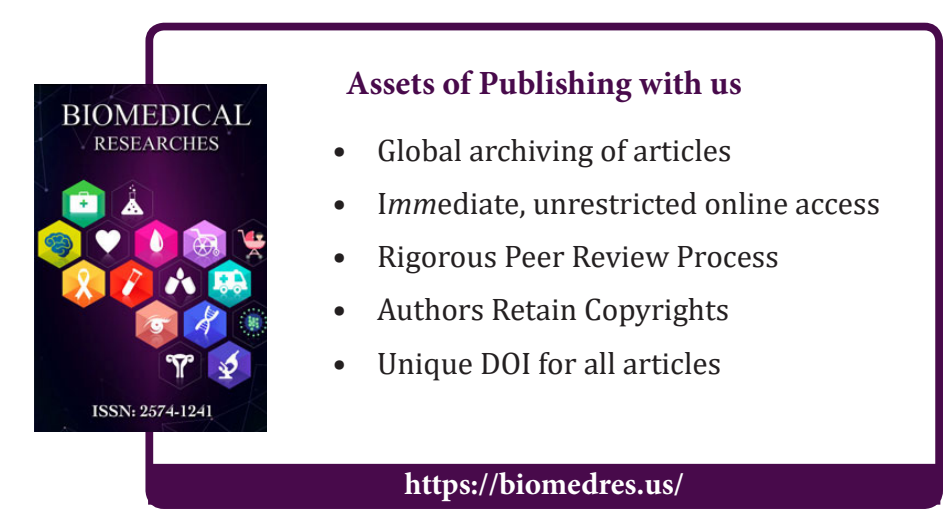

Cite this article: Asghar N, Zeeshan A, Shama M, Kahkashan I, Zahra H. Prenatal Diagnosis of Dystrophin Gene Mutations using Multiplex Ligation Dependent Probe Amplification (MLPA) for Duchene Muscular Dystrophy. Biomed J Sci \& Tech Res 12(3)-2018. BJSTR. MS.ID.002251. DOI: $10.26717 /$ BJSTR.2018.12.002251. 